\section{Clinical Significance of Severe Hyper- tension in the Field: Is it Rational to Treat Severe Hypertension in the Field?}

\author{
*Steven A. Meador, MD, MPH, FACEP, Richard \\ C. Wuerz, MD, Gregory E. Swope, EMT-P \\ Center for Emergency Medicine, University Hospital, \\ The Milton S. Hershey Medical Center, The Pennsylvania \\ State University, Hershey, Pennsylvania
}

Hypothesis: Prehospital antihypertensive therapy may benefit patients with severe hypertension (defined as systolic blood pressure [SBP] $>200 \mathrm{mmHg}$ ) and altered consciousness.

Participants: One-year (1992) series of 2,553 nonhypotensive (SBP $>100 \mathrm{mmHg}$ ), advanced life support (ALS) patients without trauma and with known final diagnoses and outcomes.

Setting: University-based ALS system in a rural area.

Methods: ALS treatments based on regional protocol and physician on-line direction. Hospital diagnoses and outcomes of patients with severe hypertension $(n=164)$ were studied.

Results: Of all the ALS patients without trauma, $7.4 \%$ $(203 / 2,758)$ were excluded due to inadequate follow-up. Cases with severe hypertension constituted $6 \%$ (164/2,553) of remaining ALS transports. Hospital mortality was $4.3 \%(7 / 164)$, while for normotensive patients it was $5.3 \%\left(126 / 2,389 ; \chi^{2}=0.31: p=0.575\right)$. Only 36 cases with severe hypertension had abnormal Glasgow Coma Scale scores (GCS $<15$ ); six had intracranial hemorrhages, while seven had hypoglycemic events, and four experienced respiratory failure.

Conclusions: Severe hypertension, even with altered consciousness, is a clinical finding of little clinical significance. The prehospital use of antihypertensive agents is unlikely to be beneficial.

\section{Emergency Medical Services Collisions in an Urban System}

\author{
*William Biggers, $M D,{ }^{1}$ Brian Zachariah, $M D^{2}$ \\ Paul Pepe, $\mathrm{MD}^{2}$ \\ 1. University of Texas-Houston, Emergency Medicine \\ Residency \\ 2. Houston Fire Department, Division of Emergency Medical \\ Services, Houston, Texas
}

Objectives: Determine the incidence, severity and contributing factors of emergency medical services collisions (EMSC).

Design: Comprehensive review of all EMSC records for calendar year 1993.

Setting: Urban emergency medical services (EMS) system serving a population of 2-million and logging 2,651,760 miles/year.

Inclusion criteria: All collisions involving vehicles assigned to the EMS division of the fire department. Major collisions were defined as those resulting in transport of a patient to the hospital or damage assessed as major by the review board.

Results: There were 86 collisions identified in 1993 , yielding a collision rate of $3.2 / 100,000$ miles driven. Seventy-four $(86 \%)$ of the records were available for analysis. There were eight (10.8\%) major EMSC with 17 victims transported to the hospital. Drivers with prior EMSC accounted for $23 / 74(31.1 \%)$ collisions and significantly more major collisions $(5 / 8, p<0.05)$. These same drivers also produced most of the injuries $(15 / 17$, $p<0.05)$. There were no reported fatalities. Lights-andsiren travel represented $52.7 \%$ of EMSC ( $p=$ NS). Most $(67 / 74,90.5 \%)$ EMSC were ruled nonpreventable by the review board. The most common mechanism was backing the vehicle $(16 / 74,21.6 \%)$. Few collisions occurred at intersections $(10 / 74,13.5 \%) ; 3 / 10(30 \%)$ of these were major accidents.

Conclusions: These findings contradict previously published studies that suggested higher rates of collisions at intersections. Use of lights and siren was not associated with EMSC in this system. Drivers with prior EMSC accounted for a disproportionate number of major collisions and victims. A standardized format for EMSC reporting needs to be developed that would allow comparison between systems. 